Separata aus Verhaltenstherapie 2, Band 4, 1994 


\title{
Sind psychische Störungen in den neuen Bundesländern häufiger?
}

\author{
Are Mental Disorders More Frequent in Eastern Germany?
}

\author{
H.-U. Wittchen ${ }^{a}$, G. Lachner ${ }^{a}$, A. Perkonigg ${ }^{a}$, J. Hoeltz ${ }^{b}$ \\ ${ }^{a}$ Max-Planck-Institut für Psychiatrie, Klinisches Institut, Klinische Psychologie \\ ${ }^{\mathrm{b}}$ Infratest Gesundheitsforschung, München
}

\begin{abstract}
Zusammenfassung und Schlüsselwörter
Im Rahmen einer Reanalyse von vier Erhebungen an jugendlichen und erwachsenen Probanden in den neuen und alten Bundesländern in den Jahren 1990 bis 1991/92 wurden Angaben zu Indikatoren für Depressivität, Angst/psychosomatische Beschwerden und Substanzgebrauch in Ost- und Westdeutschland verglichen. Zum Zeitpunkt der Untersuchungen gab es im Gegensatz zu der von einzelnen Autoren angeführten Erwartung keine eindeutigen Hinweise auf eine insgesamt erhöhte psychiatrische Morbidität in den neuen Bundesländern. Depressive Symptome und der Gebrauch von illegalen Drogen und Medikamenten sind durchgängig in den alten Bundesländern häufiger nachzuweisen als in den neuen Bundesländern. Allenfalls ergaben sich für die Stichproben Ost für einzelne depressionstypische sowie einzelne psychovegetative Symptome und für Alkoholgebrauch statistisch signifikant erhöhte Werte. Da keine Diagnosekriterien erhoben wurden, muß die Aussagekraft der Daten dahingehend eingeschränkt werden, daß lediglich Indikatoren für psychische Auffälligkeiten erhoben wurden.
\end{abstract}

Psychische Störungen · Angst - Depression - Substanzmißbrauch Ost- und Westdeutschland, Vergleich

\begin{abstract}
Summary and Key Words
Indicators for tendency to depression, anxiety, psychosomatic complaints and substance abuse, collected in four independent surveys of the East and West German population, are compared to test the hypotheses of an elevated prevalence of mental disorders in Eastern Germany after the unification. Contrary to expectations of several authors we found indications for a higher morbidity in the West German samples, especially with regard to depressive symptoms and substance abuse (illegal drugs). Only for young adults indications were found for some variables, that might point to a higher prevalence of psychosomatic complaints and selected indicators for depression. The paper discusses several critical issues that make it difficult to estimate to what degree a real increase in the prevalence of mental disorders in Eastern Germany has occurred after the unification process. Among these the general lack of studies using diagnostic instruments to estimate the prevalence of specific mental disorders is highlighted.
\end{abstract}

Prevalence of psychosomatic complaints · Anxiety · Depression Substance abuse - Comparison of Eastern and Western Germany

\section{Einleitung}

In einer Reihe zumeist populärwissenschaftlicher Veröffentlichungen [z. B. Degen, 1989] wurde in den letzten Jahren darauf hingewiesen, daß in der Folge der Wiedervereinigung psychische Probleme in den neuen Bundesländern stark ansteigen. Dabei ist auffällig, daß sich diese Aussagen weniger auf Daten als vielmehr auf einzelne Beobachtungen stützen, die kaum oder nur lückenhaft durch empirische Daten belegt sind. Keine Untersuchung hat sich bislang direkt mit der Prävalenz und Inzidenzermittlung spezifischer Formen psychischer Störungen in den neuen Bundesländern (BL) befaßt. Zwar liegen eine Reihe von Fragebogen- und Interviewstudien aus der Zeit nach der Wiedervereinigung vor, die sich mit der Beschreibung der Korrelate veränderter Lebensbedingungen in den neuen BL auf der Grundlage soziologischer und/oder psychologischer Konstrukte beschäftigt haben [Oettingen et al., 1988; Allensbacher, Archiv, 1989, 1990; Benken et al., 1990; Dehlinger und Ortmann, 1992; Hänsgen et al., 1991; Kirschner, 1993; Kasielke et al., 1993; Mummendey, 1994], jedoch erlauben sie weder gesicherte Aussagen über die Prävalenz und über die Inzidenz psychischer Störungen (z.B. von Angststörungen, somatoformen Störungen, depressiven Störungen, Abhängigkeitserkrankun- gen) nach dem Kriterienkatalog der ICD-10 (WHO, 1991) noch aufgrund ihrer methodischen Vorgehensweisen die näherungsweise Abschätzung der psychischen Morbidität. Derartige Aussagen würden erfordern, daß spezifische, auf Reliabilität und Validität geprüfte Fallfindungsinstrumente eingesetzt würden, wie z.B. das Strukturierte Klinische Interview für DSM-III-R [Wittchen et al., 1990] oder das Composite International Diagnostic Interview (CIDI) [Wittchen und Semler, 1990].

Auch indirekte Schlußfolgerungen aufgrund vorliegender repräsentativer epidemiologischer Studien in Ost- und Westdeutschland aus der Zeit vor der Wiedervereinigung helfen kaum weiter. Die einzige repräsentative bundesdeutsche $\mathrm{Be}$ völkerungsuntersuchung, in der spezifisch die Prävalenz und zum Teil die Inzidenz psychischer Störungen erhoben wurden, ist die bundesweite Münchner Follow-up-Studie aus dem Jahre 1981 [zusammenfassend Wittchen und von Zerssen, 1988; Wittchen et al., 1992]. Für den oberbayerischen Raum (Traunstein) liegen zudem die Prävalenzschätzungen von Fichter [1990] vor. Klinische PrävalenzratenStudien aus der ehemaligen DDR hingegen liegen unseres Wissens überhaupt nicht vor, so daß weder die These einer etwaigen Zunahme getestet werden kann, noch ein direkter Vergleich der alten und neuen BL möglich ist. 
Die Frage nach Unterschieden zwischen Ost und West in der psychiatrischen Morbidität ist nicht nur aus gesundheitsplanerischer Hinsicht (z.B. Aufbau von präventiven und therapeutischen Diensten sowie Etablierung problemspezifischer Präventions- und Therapieangebote) bedeutsam, sondern auch aus theoretischen Überlegungen (Ätiologie, Pathogenese, Entwicklungspsychologie). Eine Vielzahl der bisher durchgeführten sozialwissenschaftlichen Untersuchungen hat implizit oder explizit als Untersuchungshypothese, daß in den neuen BL durch die Wiedervereinigungssituation eine Vielzahl sogenannter depressogener Risikofaktoren aktiviert wurde, die sich eigentlich in einer erhöhten Prävalenz ausdrücken müßten [Oettingen et al., 1988]. Oettingen und Seligman [1990] haben in diesem Zusammenhang die Wiedervereinigung als ein experimentelles Hilflosigkeitsparadigma bezeichnet und darauf bezugnehmend die Hypothese formuliert, daß psychovegetative Einzelsymptome und insbesondere depressive Kognitionen unter den sich radikal verändernden gesellschaftlichen Bedingungen im Osten zunehmen müßten. Die wenigen kleineren sozialwissenschaftlichen Studien, die sich mit dieser Frage beschäftigt haben, erlauben jedoch aus den oben erwähnten Gründen keine Prävalenzratenabschätzungen für Depression, da sie nicht populationsbezogen durchgeführt wurden oder gar ausschließlich studentische Stichproben einbeziehen [z. B. Basten et al., im Druck]. Zudem sind die Befunde dieser Studien widersprüchlich. Drei Studien [Allensbacher Archiv, 1989, 1990; Oettingen und Seligman, 1990; Piel, 1991) geben Hinweise auf eine erhöhte Morbidität, während andere [Dehlinger und Ortmann, 1992; Dahlbert, 1993; Baier, 1992] eher ein Gleichbleiben oder sogar niedrigere Raten in den neuen BL aufzeigen. Die dabei berücksichtigten Indikationen sind allerdings insgesamt durch eine große «psychpathologische Armut» gekennzeichnet. Sie beschränken sich zumeist auf ad hoc entwickelte, mangelhaft auf Reliabilität oder Validität geprüfte, unspezifische Depressionssymptome, somatische Beschwerden sowie konstruktbezogene Aussagen zu Social support und Lebenszufriedenheit, ohne zudem eine klare Trennung zwischen den einzelnen Ebenen zu ermöglichen.

\section{Fragestellungen}

In dieser unbefriedigenden Ausgangslage haben wir uns entschlossen, eine Reanalyse vier vorliegender Datensätze durchzuführen. Dabei handelt es sich um unabhängige Surveys, die weitgehend durch gleichartige Fragen und ähnliche Untersuchungsinstrumente charakterisiert sind. Zudem wurden sie alle relativ zeitgleich kurz nach der Wiedervereinigung durchgeführt. Zwar hat keine der vier Untersuchungen spezifische Fallfindungsmethoden für psychische Störungen verwendet, jedoch erlauben alle zumindest die Ableitung einiger methodisch gut abgesicherter Indikatorvariablen für psychische Störungen, die eine Prüfung folgender Fragestellungen erlauben:

1. Lassen sich im Vergleich mit den alten BL bei Erwachsenen und bei Jugendlichen in den neuen BL häufiger bzw. stärker ausgeprägte depressionsspezifische Symptome nachweisen?

2. Sind etwaige Unterschiede abhängig von depressionstypischen Risikofaktoren wie Alter, Geschlecht, Familienstand und Berufstätigkeit?

3. Lassen sich im Vergleich mit den alten BL bei Erwachsenen und Jugendlichen häufiger bzw. stärker ausgeprägte "psychosomatische» Beschwerden feststellen?

4. Ergeben sich zwischen den alten und neuen BL Unterschiede bezüglich des Gebrauchs psychotroper Substanzen (Alkohol, illegale Drogen, Medikamente)?

\begin{abstract}
Methodik
Grundlage unserer Arbeit ist die Reanalyse der in Tabelle 1 angegebenen Untersuchungen, die alle 1-3 Jahre nach der Wiedervereinigung durchgeführt wurden. Das Untersuchungsinstrumentarium bestand aus einem Fragebogen, der den Probanden postalisch zugestellt wurde. Neben Fragen zu Soziodemographie, Einstellungen und Gesundheitsverhaltensweisen wurde insbesondere die auf Reliabilität und Validität geprüfte psychopathologische Selbstbeurteilungsskala Beschwerdenliste (B-L-Skala) [von Zerssen, 1976a, b] eingesetzt, aus der Indikatoren für die Bereiche Depression sowie Angst- und psychosomatische Probleme herausgegriffen wurden. Aufgrund leicht
\end{abstract}

Tab. 1. Datenquellen

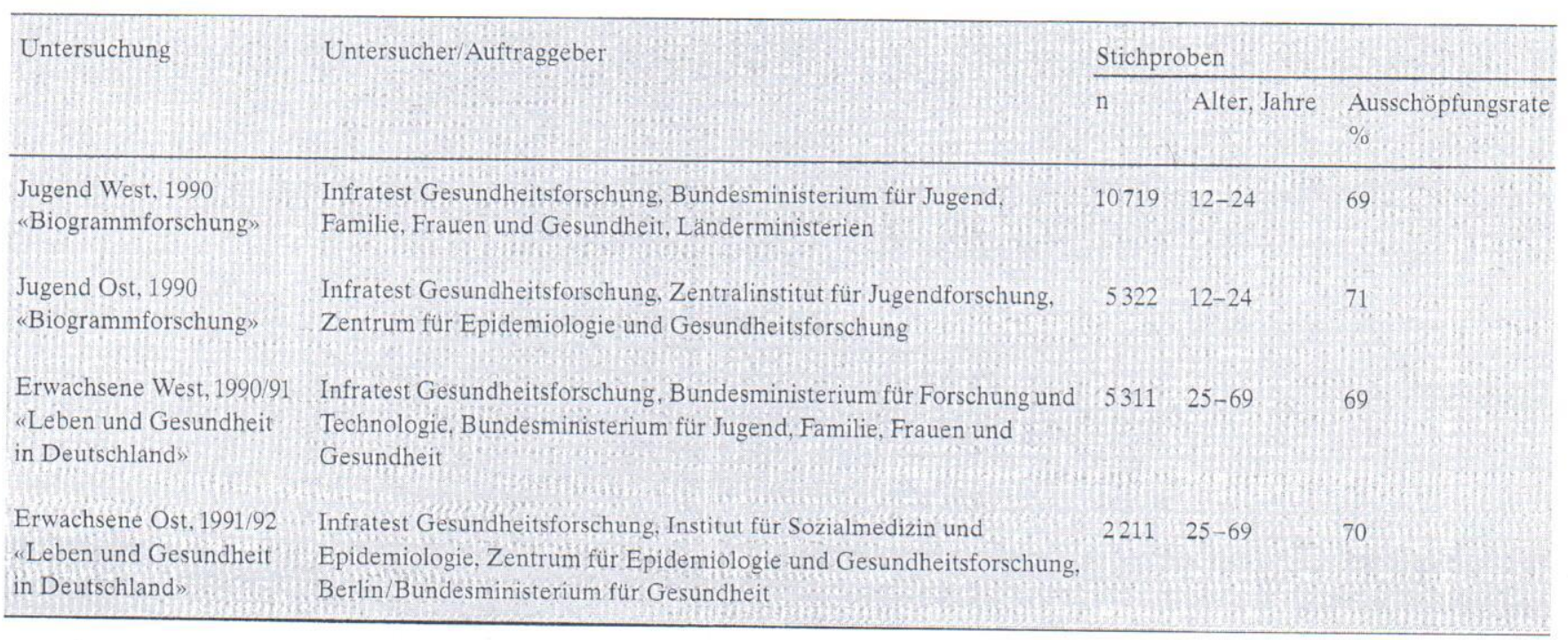


veränderter Skalierungsvorgaben haben wir wegen der Gefahr artifizieller, rein methodisch bedingter Unterschiede darauf verzichtet, die standardisierten Skalensummenwerte der B-L-Skala zu berechnen. Im einzelnen haben wir die in Tabelle 2 dargestellten Indikatorvariablen ausgewählt. Neben den oben genannten Bereichen analysierten wir die Angaben für die Bereiche Substanzgebrauch und -mißbrauch (Alkohol und illegale Drogen) sowie für die aktuelle Einnahme an ausgewählten Psychopharmakagruppen (Schlafmittel, Beruhigungsmittel, andere Psychopharmaka).

Die im folgenden angeführten Ergebnisse beruhen jeweilig auf Zahlen, die auf die gewichtete Grundgesamtheit bezogen sind, um mögliche systematische Verzerrungen durch Ausfälle zu kompensieren. Alle Stichprobengrößen beziehen sich auf ungewichtete Stichproben. Alle Signifikanzprüfungen alte versus neue BL wurden mittels Chi-Quadrat-Test zweiseitig vorgenommen.

\section{Ergebnisse}

\section{Unterschiede in den Depressivitätsindikatoren?}

Bezüglich der Indikatorvariablen für Depressivität (Abb. 1) ergeben sich bei Erwachsenen für die neuen BL fast durchgängig signifikant seltenere Angaben «starker» Beschwerden für die Variablen Schlafstörungen, Grübeln, Mattigkeit und innere Unruhe.

Beim Vergleich der beiden Jugendlichen-Stichproben (Abb. 2) zeigte sich ein etwas differenzierteres Bild. Aber auch hier sind in den alten BL etwas höhere Werte für traurige Verstimmung (alte BL $12,8 \%$ vs. neue BL $11,7 \%$, nicht signifikant) und hochsignifikant häufigere Angaben bezüglich Suizidtendenzen zu erkennen (alte BL $19,2 \%$ vs. neue BL $\left.9,7 \%, \chi^{2}=226,59 ; \mathrm{p}<0,0001\right)$. Appetitlosigkeit, Erschöpfung und Schlafstörungen wurden hingegen in der JugendlichenStichprobe der neuen BL hochsignifikant häufiger (alle $\mathrm{p}<0,0001)$ berichtet.

Unterschiede in Depressivitätsindikatoren in Abhängigkeit von ausgewählten soziodemographischen Risikofaktoren?

Zur Überprüfung möglicher modifizierender Variablen wurden die Depressivitätsindikatoren der Jugendlichen-Stichprobe geschlechtsspezifisch ausgewertet (siehe Abb. 2). Erwartungsgemäß bestätigte sich in allen Stichproben der Be-

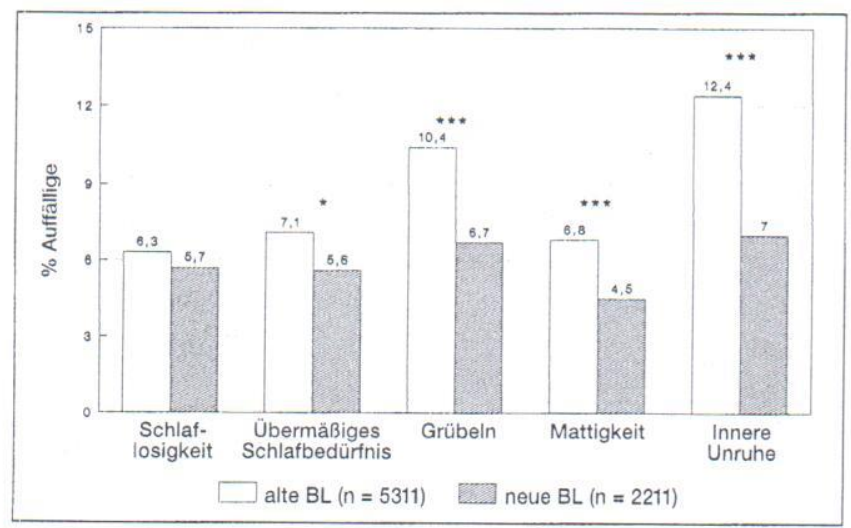

Abb. 1. Indikatoren für depressive Störungen bei Erwachsenen. Mittlere Häufigkeiten von Angaben «stark» aus «stark», «mäßig», «kaum», «gar nicht» bezüglich der Frage: «Wie stark leiden Sie unter folgenden Beschwerden?» Die Zahlen sind Prozentzahlen aus den gewichteten Stichproben alte und neue Bundesländer (BL). Signifikanzprüfungen alte versus neue $\mathrm{BL}:{ }^{*} \mathrm{p}<0,05 ; * * \mathrm{p}<0,01 ; * * \mathrm{p}<0,001$.
Tab. 2. Indikatorvariablen für psychische Störungen

\begin{tabular}{ll}
\hline Konstrukt & Indikatoren \\
\hline Depression & $\begin{array}{l}\text { Beschwerdenliste (BL), } \\
\text { [nach von Zerssen, 1976a, b] } \\
\text { Appetitlosigkeit } \\
\text { Schlafstörungen/Schlaflosigkeit } \\
\text { Erschöpfung } \\
\text { Unruhe, Erregbarkeit, Neryosität } \\
\text { Traurige Verstimmung }\end{array}$ \\
& $\begin{array}{l}\text { Suizidtendenzen } \\
\text { Angst und Psychosomatik }\end{array}$ \\
& $\begin{array}{l}\text { Einzelbeschwerden der Beschwerden- } \\
\text { liste (BL), [nach von Zerssen, 1976a, b] } \\
\text { Gesamtzahl an Beschwerden }\end{array}$ \\
Substanzgebrauch & $\begin{array}{l}\text { Alkohol } \\
\text { Drogen } \\
\text { Medikamente }\end{array}$ \\
\hline BL = Bundesländer. & \\
\hline
\end{tabular}

fund höherer Werte für weibliche Probanden. Weiter zeigten sich bei den Frauen stärkere Differenzen zwischen alten und neuen $\mathrm{BL}$ bezüglich häufiger Appetitlosigkeit, Erschöpfung und Schlafstörungen. Häufige traurige Verstimmung wiederum gaben die weiblichen jugendlichen Probandinnen Ost signifikant seltener an als die Vergleichsgruppe der alten BL. Selbstmordgedanken wurden sowohl von weiblichen wie männlichen Jugendlichen der neue BL hochsignifikant seltener berichtet als in den alten BL.

Bezüglich des Risikofaktors Berufstätigkeit fand sich für die Indikatorvariable «traurige Verstimmung» kein signifikanter Unterschied zwischen den neuen und alten BL, ebensowenig für den Risikofaktor Familienstand.

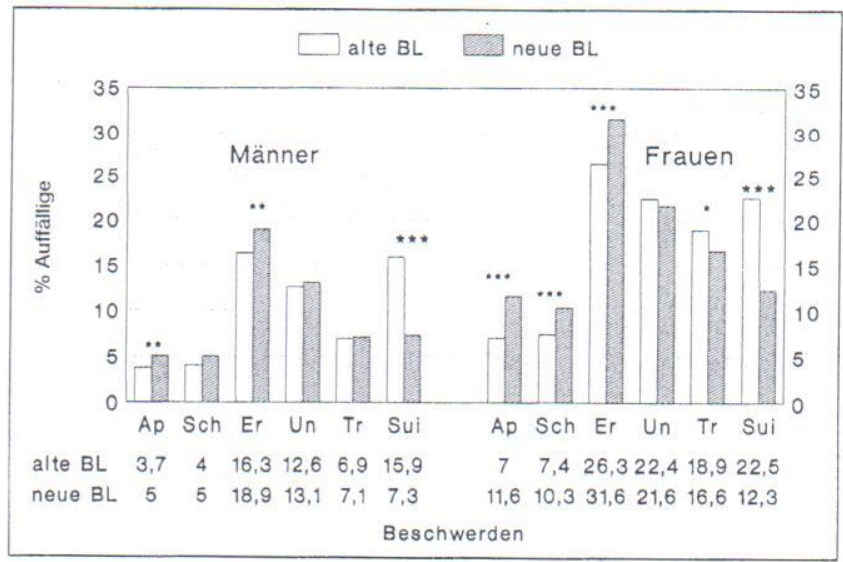

Abb. 2. Indikatoren für depressive Störungen bei Jugendlichen nach Geschlecht. Mittlere Häufigkeiten von Angaben «häufig» aus «häufig», «selten», «nie» bezüglich der Frage: «Wie häufig haben Sie in den letzten 12 Monaten folgende Beschwerden gehabt?»(Ap = Appetitlosigkeit, $\mathrm{Sch}=$ Schlafstörungen/Schlaflosigkeit, $\mathrm{Er}=$ Erschöpfung, Un = Unruhe, Erregbarkeit, Nervosität, $\mathrm{Tr}=$ traurige Verstimmung). Die Angaben zu «Sui» beziehen sich auf Angaben von «ja, häufiger» aus «nein», «ja, einmal» oder «ja, häufiger» auf die Frage: «Hatten Sie schon einmal Selbstmordgedanken?» Die Zahlen sind Prozentzahlen aus den gewichteten Stichproben «Männer, alte $\mathrm{BL}$ » $(\mathrm{n}=5358)$, «Männer, neue $\mathrm{BL}$ » $(\mathrm{n}=2528)$, «Frauen, alte $\mathrm{BL}$ » $(\mathrm{n}=5361)$ und Frauen, neue $B L »(n=2794)$. Signifikanzprüfungen alte vs. neue BL: $* 0<0,05: * * 0<0.01: * * * \mathrm{p}<0.001$. 


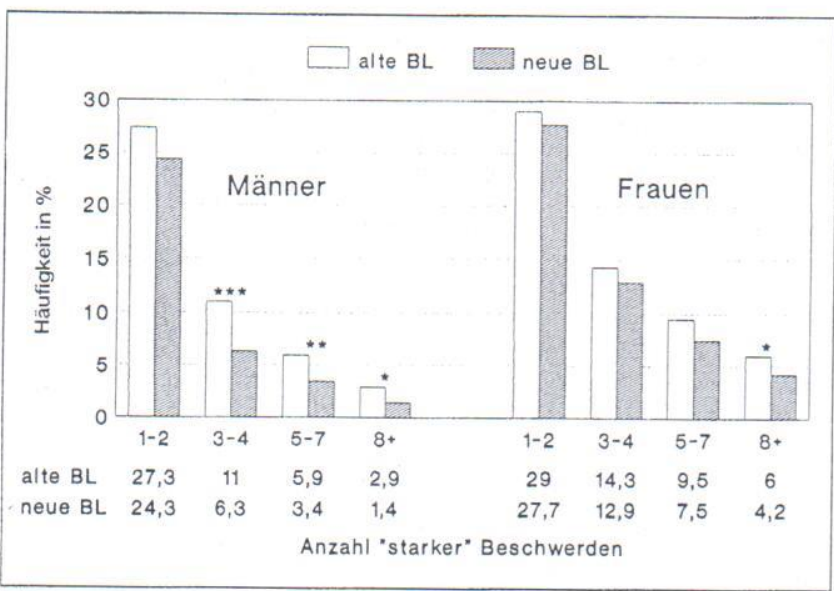

Abb. 3. Anzahl starker Beschwerden bei Erwachsenen nach Geschlecht. Mittlere Häufigkeiten von Angaben «stark» aus «stark», «kaum», "gar nicht» bezüglich der Frage: «Wie stark leiden Sie unter folgenden Beschwerden?» Die Zahlen sind Prozentzahlen aus den gewichteten Stichproben «Männer, alte BL» $(n=2623)$, «Männer, neue BL» $(n=1051)$, «Frauen, alte BL» $(n=2688)$ und «Frauen, neue $B L »(n=1160)$. Signifikanzprüfungen alte versus neue BL: ${ }^{*} \mathrm{p}<0,05 ; * * \mathrm{p}<0,01 ; * * * \mathrm{p}<0,001$.

\section{Unterschiede in psychosomatischen Beschwerden?}

Der Symptomhäufigkeitsvergleich der beiden ErwachsenenStichproben ergibt für die alten BL insgesamt eine signifikant höhere Beschwerdenbelastung $\left(\chi^{2}=58,48, \mathrm{df}=4, \mathrm{p}<0,0001\right)$. Wie aus Abbildung $3 \mathrm{zu}$ ersehen ist, liegen sowohl bei Männern wie auch bei Frauen die alten BL bezüglich Anzahl und Häufigkeit der als gravierend geschilderten Beschwerden in allen Kategorien über den neuen BL. Gleichzeitig sehen wir den bereits bekannten Befund, daß Frauen deutlich häufiger angeben, unter psychovegetativen und sonstigen Beschwerden zu leiden, als Männer.

Bei den Jugendlichen-Stichproben ergibt sich ein deutlich abweichendes Bild (Abb. 4). Sowohl einzelne Beschwerden wie auch der Gesamtwert ist in der Stichprobe der neuen BL signifikant erhöht $\left(\chi^{2}=23,72, \mathrm{df}=4, \mathrm{p}<0,0001\right)$. Abbildung 4 läßt erkennen, daß in den neuen Bundesländern signifikant häufiger die Merkmale Appetitlosigkeit, Herzklopfen, Schlafstörungen, Schweißausbrüche, Vergeßlichkeit und Erschöpfung angegeben werden.

Unterschiede in Indikatoren für Angst bzw. psychosomatische Beschwerden in Abhängigkeit von ausgewählten soziodemographischen Risikofaktoren?

Der Eindruck einer erhöhten psychosomatischen Beschwerdenlage mit einem Schwerpunkt auf Symptomen, die dem Angstbeschwerden-Komplex sowie unspezifischen somatischen Erschöpfungssyndromen zuzuordnen sind, bestätigt sich auch bei der nach Geschlecht differenzierten Analyse nach Anzahl der Beschwerden. Während Erwachsene in den neuen BL also eine eher niedrigere Symptomhäufigkeit aufweisen, zeigen Jugendliche eine zum Teil deutlich erhöhte Beschwerdenlage. Die Differenz neue versus alte BL, die in Abbildung 5 graphisch dargestellt ist, ist für die Gesamtzahl an Beschwerden bei den jugendlichen Männern $\left(\chi^{2}=20,73\right.$, $\mathrm{df}=4, \mathrm{p}=0,0004$ ) ausgeprägter als bei den jugendlichen Frauen $\left(\chi^{2}=12,76, d f=4, p=0,0125\right)$. In der Erwachsenen-Stichprobe fand sich daruber hinaus eine stetige Zunahme der

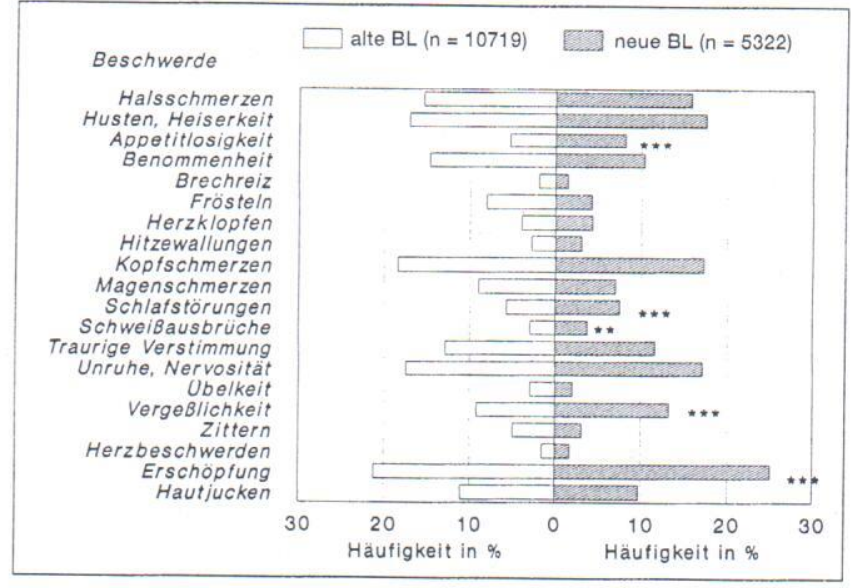

Abb. 4. Indikatoren für Angst und psychosomatische Beschwerden bei Jugendlichen. Mittlere Häufigkeiten von Angaben «häufig» aus «häufig», «selten», «nie» bezüglich der Frage: «Wie häufig haben Sic in den letzten 12 Monaten folgende Beschwerden gehabt?»

Die Zahlen sind Prozentzahlen aus den gewichteten Stichproben. Signifikanzprüfungen alte versus neue $\mathrm{BL}:{ }^{*} \mathrm{p}<0,05 ;{ }^{*} \mathrm{p}<0,01$; $* * * \mathrm{p}<0,001$.

Beschwerdenhäufigkeit mit höherem Alter. Gravierend erhöht war ferner die Beschwerdenhäufigkeit bei Probanden, die getrennt, verwitwet oder geschieden waren. In der Jugendlichen-Stichprobe konnte dies aufgrund zu geringer Fallzahlen nicht geprüft werden. Auffällig war, daß Arbeitslosigkeit bei Erwachsenen nur in den alten BL mit einer deutlich erhöhten psychosomatischen Beschwerdenlage assoziiert war, nicht jedoch in den neuen.

\section{Unterschiede im Substanzgebrauch?}

Zur Erfassung von Indikatoren zum Substanzmißbrauch ist einleitend zu sagen, daß keine klassifikatorisch-diagnostische Beurteilung von Abhängigkeit oder gar Mißbrauchsformen möglich ist, da in den vier Studien zwar höchst differenziert Häufigkeit und Menge des Konsums dokumentiert sind, aber die notwendigen Fragen zur Beurteilung von Störungen fast vollständig fehlen. Aus diesem Grund können wiederum nur einzelne grobe Indikatoren ausgewählt werden.

Alkohol. Einer der Indikatoren betrifft den Anteil der befragten Erwachsenen, die täglich alkoholische Getränke wie Bier, Wein und Spirituosen zu sich nehmen. Tabelle 3 veranschaulicht deutliche Unterschiede zwischen alten und neuen BL hinsichtlich des Konsums von Bier. So geben über $40 \%$ der befragten erwachsenen Männer in den neuen BL an, täglich oder fast täglich Bier zu konsumieren, in den alten BL hingegen unter $20 \%$ der erwachsenen Männer $\left(\chi^{2}=180,72\right.$, $\mathrm{df}=1, \mathrm{p}<0,0001)$. In den alten $\mathrm{BL}$ wiederum wird - in allerdings erheblich geringerem Umfang - häufiger Wein getrunken (für die Männer $\chi^{2}=16,25, \mathrm{df}=1, \mathrm{p}<0,0001$ ). Bezüglich hochprozentiger alkoholischer Getränke findet sich kein signifikanter Unterschied für die Männer $\left(\chi^{2}=1,22\right.$, $\mathrm{df}=1, \mathrm{p}=0,269)$. Insgesamt deutet sich an, $\mathrm{da} \beta$ in den neuen $\mathrm{BL}$ häufiger Alkoholika täglich oder fast täglich getrunken werden. Bei Frauen zeigen sich keine signifikanten Unterschiede mit Ausnahme eines niedrigeren Anteils von Weinkonsumentinnen $\left(\chi^{2}=5,41, \mathrm{df}=1, \mathrm{p}=0,020\right)$ in den neuen BL. Die Frage nach einem Gefühl subjektiver Abhängigkeit von 
Alkohol wie erstaunlicherweise auch von Rauschmitteln wird sowohl von jugendlichen Männern (allerdings nicht signifikant) wie auch jugendlichen Frauen (jeweils $\chi^{2}=5,203$, $\mathrm{df}=1, \mathrm{p}=0,0225)$ in den neuen $\mathrm{BL}$ häufiger bejaht als in den alten.

Illegale Drogen. Psychotrope Substanzen wurden, wie Tabelle 4 zeigt, zum Zeitpunkt der Untersuchungen wesentlich öfter in den alten BL konsumiert. Dabei sind die häufigsten illegal konsumierten Drogen Marihuana und Haschisch mit $12,3 \%$ in der West-Stichprobe im Vergleich zu weniger als $0,9 \%$ in der Ost-Stichprobe. Zudem ist zu berücksichtigen, daß in der Ost-Stichprobe die überwiegende Mehrzahl erst ein- oder zweimal Probierverhalten gezeigt hat, während im Westen die regelmäßigen und unregelmäßigen Nutzer die größte Gruppe ausmachen. Neben Marihuana und Haschisch ist in den alten BL auf die Häufigkeit von Kokain mit 1\% und Tranquilizern mit zusammen ungefähr $1 \%$ hinzuweisen. Zusammenfassend finden sich somit einige diskrete Hinweise für eine erhöhte Rate von Alkoholmißbrauch und -abhängigkeit in den neuen BL, während zumindest bei Jugendlichen der neuen BL die Raten für Rauschmittelmißbrauch und -abhängigkeit zum Untersuchungszeitpunkt durchgängig niedriger liegen.

Psychopharmaka. Zwischen 83 und 97\% der Erwachsenen in den neuen und $73-80 \%$ in den alten BL gaben an, «noch nie» Psychopharmaka vom Typ der Beruhigungs-, Schlafmittel bzw. andere Psychopharmaka eingenommen zu haben. In den neuen BL berichtet ein wesentlich höherer Prozentsatz von Männern (jeweils $\mathrm{p}<0,001$ ) und ein erhöhter Prozentsatz der untersuchten Frauen für Schlafmittel $\left(\chi^{2}=7,62, \mathrm{df}=1\right.$, $\mathrm{p}=0,0058)$ und andere Psychopharmaka $\left(\chi^{2}=79,76, \mathrm{df}=1\right.$, $\mathrm{p}<0,0001$, noch nie derartige Medikamente konsumiert $\mathrm{zu}$ haben.

Wie Abbildung 6 veranschaulicht, konsumieren Frauen 2- bis 3 mal so häufig Psychopharmaka als Männer. Dabei stehen Beruhigungsmittel unterschiedlichster Form an erster Stelle. Fast $4 \%$ der Frauen in den alten und neuen BL geben an,

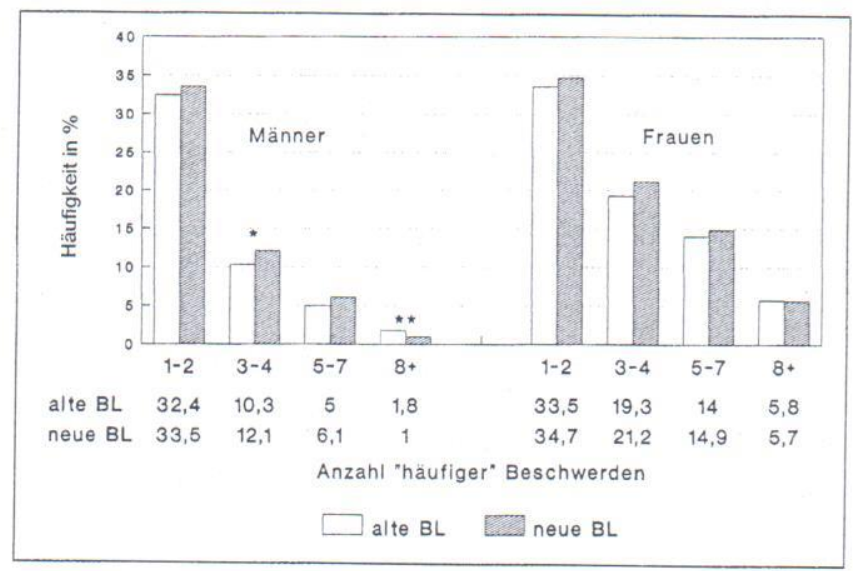

Abb. 5. Anzahl häufiger Beschwerden bei Jugendlichen nach Geschlecht. Mittlere Häufigkeiten von Angaben «häufig» aus «häufig», «selten», «nie» bezüglich der Frage: «Wie häufig haben Sie in den letzten Monaten folgende Beschwerden gehabt? » Die Zahlen sind Prozentzahlen aus den gewichteten Stichproben «Männer, alte BL» $(\mathrm{n}=5358)$, «Männer, neue BL» $(\mathrm{n}=2528)$, «Frauen, alte BL» $(\mathrm{n}=5361)$ und "Frauen, neue $B L »(n=2794)$. Signifikanzprüfungen alte versus neue $B L: * p<0,05 ; * * p<0,01 ; * * * p<0,001$. täglich oder mehrmals pro Woche derartige Medikamente einzunehmen. Direkt danach folgen bei Frauen Schlafmittel und Psychopharmaka. Zwischen alten und neuen BL fanden sich weder bei Frauen noch bei Männern signifikante Unterschiede bezüglich der Häufigkeit von Angaben täglich oder mehrmals wöchentlich eingenommener Psychopharmaka.

\section{Diskussion}

Vor einer zusammenfassenden Diskussion muß noch einmal auf die Begrenzungen unserer Analyse hingewiesen werden: Mangelnde diagnostische Spezifität. Ein wesentliches Manko auch unserer Studie ist, daß keine Aussagen über das Vorliegen bestimmter psychischer Störungen in den neuen und alten BL möglich sind. Der Vorteil unserer Analyse ist, daß zumindest gut untersuchte Standardskalen eingesetzt wurden. Unabhängig davon ist die mangelnde Spezifität der diagnoserelevanten Informationen gravierend zu nennen und bedarf dringend einer Verbesserung.

Kompatibilität der vier Datensätze. Trotz zum Teil identischer Fragenbereiche unterscheiden sich die Erhebungen der Jugendlichen und Erwachsenen zum Teil durch unterschiedliche Frageformulierungen oder unterschiedliche Zeitfensterdefinitionen zur Erfassung einzelner Symptome. Aus diesem Grund ist es zumeist nicht gerechtfertigt, die Ergebnisse der Erwachsenen und der Jugendlichen direkt in Beziehung zu setzen. Hinzuweisen ist auch darauf, daß die vier Erhebungen nicht vollständig zeitgleich durchgeführt wurden und sich so auf leicht unterschiedliche Zeiträume nach der Wiedervereinigung beziehen.

Statistische Analyse. Bislang haben wir lediglich Einzelmerkmale untersucht und wegen der oben erwähnten Probleme nicht versucht, über die Kombination von Einzelmerkmalen

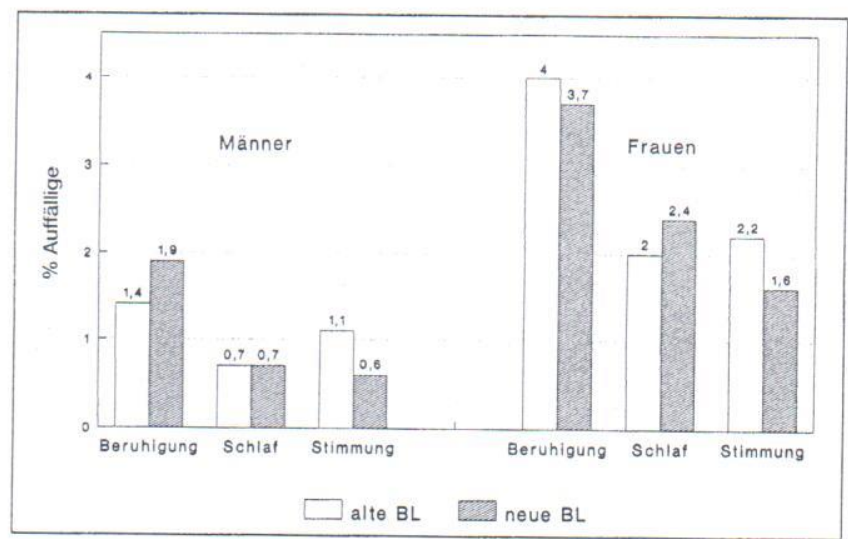

Abb. 6. Einnahme von Psychopharmaka bei Erwachsenen nach Geschlecht. Summe der Häufigkeiten von Angaben «täglich» und "mehrmals in der Woche» aus «täglich», «mehrmals in der Woche», «1-2mal in der Woche», «1-3mal im Monat», «selten» sowie «nie» und «häufig» aus «häufig», «selten», «nie» bezüglich der Frage: «Wie häufig nehmen Sie die folgenden Medikamente ein?» (Beruhigung = Beruhigungsmittel, Schlaf $=$ Schlafmittel, Stimmung = stimmungsbeeinflussende Mittel, Psychopharmaka). Die Zahlen sind Prozentzahlen aus den gewichteten Stichproben «Männer, alte BL» ( $\mathrm{n}=2623)$, «Männer, neue BL» $(\mathrm{n}=1051)$, «Frauen, alte BL» $(\mathrm{n}=2688)$ und Frauen, neue BL» $(\mathrm{n}=1160)$. Keine der Signifikanzprüfungen alte versus neue $B L$ erzielte $p<0,05$, 
Tab. 3. Alkoholgebrauch Erwachsener, gewichtete Prozentangaben. Häufigkeit der Angabe «fast täglich» aus «fast täglich», «mehrmals in der Woche», «etwa $1 \mathrm{mal}$ in der Woche», «2-3mal im Monat», «max. 1 mal im Monat», «nie»

\begin{tabular}{|c|c|c|c|c|c|c|}
\hline \multirow{2}{*}{$\begin{array}{l}\text { Variable } \\
\text { and }\end{array}$} & \multicolumn{2}{|l|}{ Männer } & \multicolumn{2}{|l|}{ Frauen } & \multicolumn{2}{|l|}{ Gesamt } \\
\hline & $\begin{array}{l}\text { alte BL } \\
(\mathrm{n}=2623)\end{array}$ & $\begin{array}{l}\text { neue BL } \\
(n=1051)\end{array}$ & $\begin{array}{l}\text { alte } \mathrm{BL} \\
(\mathrm{n}=2688)\end{array}$ & $\begin{array}{l}\text { neue } B L \\
(n=1160)\end{array}$ & $\begin{array}{l}\text { alte } B L \\
(n=5311)\end{array}$ & $\begin{array}{l}\text { neue } B L \\
(n=2211)\end{array}$ \\
\hline Bier fast täglich & 18,9 & 40,6 & 3,7 & 3,6 & 11,1 & 21,5 \\
\hline $\begin{array}{l}\text { Wein/Sekt/Obstwein } \\
\text { fast täglich }\end{array}$ & 2,5 & 0,4 & 2,1 & 0,9 & 2,3 & 0,7 \\
\hline $\begin{array}{l}\text { Hochprozentige } \\
\text { alkoholische Getränke fast } \\
\text { täglich }\end{array}$ & 1,3 & 1,9 & 0,3 & 0,3 & 0,8 & 1,1 \\
\hline
\end{tabular}

Tab. 4. Gebrauch psychotroper Substanzen bei Jugendlichen, gewichtete Prozentangaben. Häufigkeit von Angaben auf die Frage: «Kreuzen Sie bitte diejenigen Mittel an, die Sie schon einmal probiert haben (nicht im Rahmen einer ärztlichen Behandlung)»

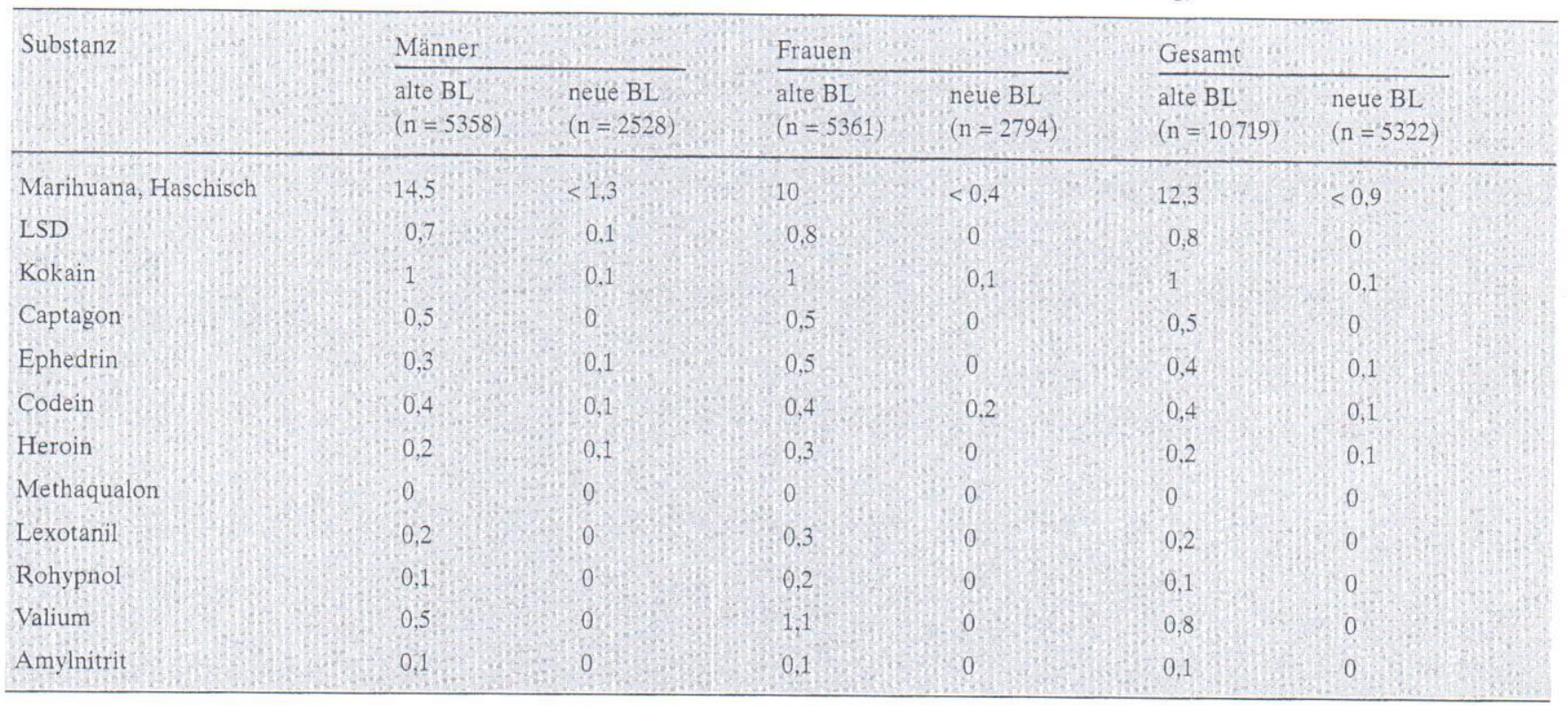

bei Anwendung komplexerer statistischer Analysemethoden spezifische Effekte zu identifizieren.

Aussagekraft. Da wir uns nur auf Querschnittsdaten beziehen, können wir zwar vergleichende Aussagen über den Status quo in Ost und West treffen, nicht aber die eingangs angesprochene Hypothese eines Anstiegs psychischer Störungen im Ostteil prüfen. So kann es sehr wohl möglich sein, daß es im Osten, verglichen mit der Situation vor der Wiedervereinigung, einen deutlichen Anstieg von einem ursprünglich niedrigeren Niveau psychopathologischer Gestörtheit gegeben hat.

Unter Berücksichtigung dieser Einschränkungen können wir zusammenfassen:

1. Es gibt zum Zeitpunkt der Untersuchungen keine eindeutigen Hinweise auf eine insgesamt erhöhte psychiatrische Morbidität in den neuen BL. Hinweise auf ein erhöhtes Auftreten psychischer Störungen ergeben sich lediglich für die Jugendlichen-Stichprobe Ost bezüglich einzelner depressionstypischer sowie psychovegetativer Symptome.

2. Im Gegensatz zu der eingangs von einzelnen Autoren angeführten Erwartung, im Osten aufgrund der besonderen gesellschaftlichen Bedingungen ein erhöhtes Ausmaß an Depressivität zu finden, fanden wir zusammenfassend eher Hinweise auf das Gegenteil. Depressive Symptome sind in den alten BL häufiger als in den neuen.

3. In ähnlicher Weise gilt dies übrigens auch für die untersuchten Indikatoren für den Mißbrauch und die Abhängigkeit von Rauschmitteln. Lediglich für Männer in den neuen BL konnte ein Hinweis auf eine höhere Alkoholabhängigkeitsgefährdung gesichert werden. In fast allen anderen Variablen, vor allem aber bezüglich der illegalen Drogen, sprechen die Gebrauchsmuster für eine vielfach höhere Prävalenz möglicher Mißbrauch- und Abhängigkeitssyndrome in den alten BL.

Diese Befundlage einer insgesamt tendentiell sogar besseren psychischen Befindenslage der Ost-Bevölkerung ist auf den ersten Blick kontraintuitiv und war von uns trotz durchaus vorliegender, eingangs zitierter analoger Befunde anderer Studien nicht erwartet worden. Was könnten die Gründe für die - überspitzt formuliert - bessere psychische Gesundheitslage der Bevölkerung in den neuen Bundesländern sein? 
Bezüglich der illegalen Drogen könnte die geringere Häufigkeit des Gebrauchs in der Ost-Stichprobe einen Status andeuten, der durch die repressivere Politik der DDR und die damit verbundene geringere Verfügbarkeit beeinflußt ist. Schwieriger fällt es, aufgrund der eingangs angeführten Überlegungen von Oettingen und Seligman [1990] zu erklären, warum keine durchgängigen depressionsspezifischen Erhöhungen zu beobachten sind. Eine Möglichkeit besteht darin, daß die Untersuchungen zu früh nach der Wiedervereinigung durchgeführt wurden. Es könnte spekuliert werden, daß die Annahmen des Hilflosigkeitsparadigmas sich erst nach längerer «Exposition» in depressionstypischen Symptomen und echter «klinischer» Morbidität niederschlagen. Hierfür könnte sprechen, daß zwar nicht bezüglich der Depressivität, sehr wohl aber für unspezifische «psychosomatische Erschöpfungssymptome» wie Appetit, Schlaf und Erschöpfung erhöhte Prävalenzen in den Ost-Stichproben nachweisbar waren. Ferner könnte der Befund, daß Frauen der neuen Bundesländer deutlichere Erhöhungen als Männer aufweisen, mit der Entwicklung korrespondieren, daß Frauen, insbesondere berufstätige Frauen, stärker von Arbeitslosigkeit und dem Zerfall der familiären Betreuungsstrukturen betroffen sind als Männer.

Möglich ist auch, daß die Ost-Untersuchungen insofern zu früh durchgeführt wurden, als daß die alten Strukturen real und mental noch durchaus vorhanden waren und so die Befragten noch nicht in vollem Umfang von der Umstellungsproblematik betroffen sind. Hierfür könnte auch sprechen, daß bei den Jugendlichen, die möglicherweise nicht wie die Erwachsenen in alte Denk- und Handlungsstrukturen eingebunden waren, durchaus diskrete Hinweise auf eine erhöhte Morbidität psychovegetativer Art gesichert werden konnten. Möglich ist ferner auch, daß Frühstadien erhöhter Morbidität durch den Befragungsmodus Fragebogen durch eventuell noch vorhandene, unrealistisch optimistische Zukunftserwartungen «überdeckt» wurden; bei einer klinisch sensibleren Interviewuntersuchung könnten somit möglicherweise andere Befunde resultieren.

Nicht vollkommen auszuschließen ist ferner die Wirksamkeit eines ostspezifischen generellen Beantwortungs-Bias. Ein solcher Response-Bias wurde in der Vergangenheit dahingehend diskutiert, daß Probanden in den alten BL eher geneigt sind, aufgrund ihrer längeren Erfahrung mit SurveyMethoden extremere Negativ-Eigenschaften anzugeben als Probanden aus den neuen BL, die eher versuchen, vorsichtigere Mittelpositionen zu beziehen. Bei unserer Überprüfung des Antwortverhaltens für verschiedene Variablenbereiche sowohl für Erwachsene als auch für Jugendliche konnten wir allerdings diesbezüglich keine systematischen Hinweise in dieser Richtung finden. Beide Stichproben zeigten z. B. bezüglich der allgemeinen Lebenszufriedenheit sowie der Beurteilung ihres gegenwärtigen Gesundheitszustandes fast identische Werte mit ähnlicher Streubreite.

Unsere kurz nach der Wiedervereinigung erhobenen Befunde scheinen zusammenfassend anzudeuten, daß allenfalls bei Jugendlichen, und hier am stärksten für arbeitslose Frauen, eine erhöhte psychische Morbidität vermutet werden kann. Vor dem Hintergrund der oben angesprochenen Hilflosigkeitshypothese ist aufgrund der Art und Ausprägung der beobachteten Symptome ferner zu vermuten, daß die Studien zum Untersuchungszeitpunkt in erster Linie Vorstadien manifester psychischer Störungen erfaßt haben. Versorgungspolitisch wäre daraus eine Intensivierung problemspezifischer präventiver Maßnahmen zur Verhinderung manifester psychischer Störungen abzuleiten.

Abschließend ist darauf hinzuweisen, daß wir keine aktuelleren Daten vorliegen haben, die eine Beurteilung der Frage erlauben, inwieweit sich zwischenzeitlich, also 2-3 Jahre nach der Erhebung, bei Erwachsenen und Jugendlichen eine Veränderung der Befundlage ergeben hat.

\section{Literatur}

Allensbacher Archiv: LfD Umfragen 5019. Allensbach, 1989. Allensbacher Archiv: LfD-Umfragen 9002. Allensbach, 1990. Baier S: Mit Empirie gegen Vorurteile: Den «typischen Ossi» gibt es nicht. Rep Psychol 1992;17:10-12.

Basten M, Florin I, Tuschen B, Wessels R, Hübner I, Bossong A, Schmidt S: Psychische Symptome und körperliche Gesundheit im vereinten Deutschland: Eine Ost-West-Vergleichsuntersuchung an einer studentischen Stichprobe. Verhaltensther Prax Forsch Perspekt (im Druck).

Benken I, Guenther C, Kabat-vel-Job O, Karig U, Keiser S, Krueger H-H, Lindner B, von Wensierski H-J, Zinnecker J: Anders und doch gleich. Jugendliche im Prozeß der Vereinigung. Eine erste gesamtdeutsche Schülerbefragung. Paed Extra Demokrat Erzieh 1990;3: $39-45$.

Dahlbert C: Psychisches Wohlbefinden und Persönlichkeit in Ost und West: Vergleich von Sozialisationseffekten in der früheren DDR und der alten BRD. Z Sozialisationsforsch Erziehungssoziol 1993;13: 82-94.

Degen R: Der real existierende Trübsinn. Psychol Heute 1989; 16:44-48.

Dehlinger E, Ortmann K: Gesundheitszufriedenheit in der Bundesrepublik Deutschland und der ehemaligen DDR - ein Vergleich. Gesundheitswesen 1992;54:88-94.

Fichter MM: Verlauf psychischer Erkrankungen in der Bevölkerung. Berlin, Springer, 1990.

Hänsgen K-D, Kasielke E, Schmidt LR, Schwenkmezger P: Persönlichkeitsvergleiche zwischen Ostdeutschen und Westdeutschen: Selbsteinschätzungen, objektive Tests und neuroserelevante Persönlichkeitsmerkmale. Trierer Psychol Ber 1991;18:1-2.

Infratest: Deutschlands Jugend in West und Ost: Einheit in den Köpfen? Gleiche Lebensziele, unterschiedliche Einstellungen. Ergebnisse einer Repräsentativ-Erhebung. Planung Analyse 1991;18: 110-112.

Kasielke E, Hänsgen K-D, Schmidt LR, Schwenkmezger P: Persönlichkeitscharakteristika ostdeutscher Bevölkerungsgruppen im Herbst 1990. Rep Psychol 1993;18:5-6.

Kirschner W, Radoschewski M: Gesundheits- und Sozialsurvey Berlin, in Senatsverwaltung für Gesundheit (Hrsg): Diskussionsbeiträge zur Gesundheits- und Sozialforschung. Berlin, Senatsverwaltung für Gesundheit, 1993.

Mummendey A: Bewältigungsstrategien «negativer sozialer Identität». Eine Begleitstudie zum ost-westdeutschen Integrationsprozeß. Antrag auf Weiterförderung eines Forschungsvorhabens durch die DFG, 1994.

Oettingen G, et al: Pessimism across cultures. Russian Judaism versus Orthodox Christianity and East versus West Berlin. Am Psychol 1988.

Oettingen G, Seligman MEP: Pessimism and behavioural signs of depression in East versus West Berlin. Eur J Soc Psychol 1990;20: $207-220$. 
Piel E: Gesundheitsreport: Deutschland Ost-West. Krankenhausarzt 1991;64:291-292.

Wittchen H-U, Essau CA, von Zerssen D, Krieg CJ, Hecht H: Lifetime and six-month prevalence of mental disorders in the Munich Follow-up Study. Eur Arch Psychiatry Clinical Neurosci 1992;241: 247-258.

Wittchen H-U, Zaudig M, Schramm E, Spengler P, Mombour W, Klug J, Horn R: SKID: Strukturiertes Klinisches Interview für DSM-III-R. Interviewheft. 2. korrigierte Aufl. Weinheim, Beltz Test, 1991.

Wittchen H-U, Semler G: Composite International Diagnostic Interview (CIDI, Version 1.0). Weinheim, Beltz, 1990.

Wittchen H-U, von Zerssen D (Hrsg): Verläufe behandelter und unbehandelter Depressionen und Angststörungen - Eine klinischpsychiatrische und epidemiologische Verlaufsuntersuchung. Berlin, Springer, 1987.
Wittchen H-U, von Zerssen D (Hrsg): Verläufe behandelter und unbehandelter Depressionen und Angststörungen - Eine klinischpsychiatrische und epidemiologische Verlaufsuntersuchung. Berlin, Springer, 1988 .

World Health Organization: International Classification of Disease. New York, 1991.

von Zerssen D: Depressivitätsskala. Weinheim, Beltz Test, 1976a.

von Zerssen D: Die Beschwerden-Liste. Weinheim, Beltz Test, 1976b.

Sonderdruckbestellungen an:

Prof. Dr. Hans-Ulrich Wittchen

Max-Planck-Institut für Psychiatrie

Klinisches Institut, Klinische Psychologie

Kraepelinstraße 2

D-80804 München (BRD) 